\title{
Les vœux du président
}

\section{Good wishes from the chairman}

\section{J.C. Goussard}

C) Springer-Verlag France 2013

Chers amis, chers lecteurs,

À l'aube de cette nouvelle année, permettez-moi avant tout, chers amis, chers lecteurs, de vous présenter au nom du bureau de l'ANMSR, tous mes vœux sincères de santé et de bonheur.

2013 sera pour notre association une année de réflexion, de prise de décision, voire de nouvelle orientation pour certaines de nos activités.

Votre association continuera d'œuvrer sans relâche et selon ses possibilités pour la promotion de notre belle spécialité, la médecine physique et de réadaptation (MPR) grâce aux actions efficaces menées de façon dévouée et totalement bénévole par ses membres actifs : mon prédécesseur Samy Bendaya, notre trésorière Béatrice Davenne, notre secrétaire Frédérique Le Breton, notre désormais ex-rédactrice en chef Véronique Salvator-Witvoet, Jacques Carzon, Jean-Luc Poindessous, Françoise Sarrazin, Martial Basta, Frédéric De Lucas Vazquez, Patricia Blondel, Olga Chapelle.

Qu'ils en soient ici solennellement remerciés.

L'ANMSR a trois pôles d'activités principaux : La Lettre de MPR, l'organisation de la Journée annuelle qui existe depuis 1987, le site et la gestion des petites annonces.

La Lettre de Médecine Physique et de de Réadaptation a été dirigée de manière rigoureuse et sans faille par le Dr Véronique Salvator-Witvoet jusqu'à la fin 2012.

À partir de 2013, chaque numéro aura son propre rédacteur en chef.

Cette Lettre a une vocation essentiellement pratique, ce qui en fait son succès. Elle constitue véritablement le véhicule de l'expérience de tous et elle est reconnue et appréciée de tous (vos fidèles abonnements en témoignent).

À l'avenir, elle doit devenir une revue de formation et d'information professionnelle.

Elle doit continuer à exister, telle est notre volonté, quelles que soient les orientations prises en fonction de l'évolution de notre spécialité et/ou de notre association. Les modalités de cette évolution seront envisagées cette année.

J.C. Goussard ( $\square)$

Paris

e-mail : jc.goussard387@orange.fr
Elle ne vivra que grâce à vous et continuera d'autant plus que vous tous, praticiens de terrain qui avez une énorme expérience, contribuerez à l'alimenter.

Merci de nous faire parvenir vos articles, ne gardez pas votre expérience pour vous, nos confrères jeunes et moins jeunes sont très intéressés par le partage des idées et de l'expérience des uns et des autres dans l'exercice de cette vaste spécialité.

Vous y trouverez également les mots croisés, fidèlement réalisés par notre ami Pierre Cassagne.

Le site de notre association, organe de communication et d'information, comporte les petites annonces et est régulièrement mis à jour. Notre ami Jacques Carzon s'y emploie pendant de nombreuses heures chaque semaine. Merci à lui.

La prochaine Journée de l'A.N.M.S.R. (la xxvII ${ }^{\mathrm{e}} \mathrm{du}$ nom) qui se déroulera le vendredi 24 mai 2013 à l'hôtel Ibis, 163 bis avenue de Clichy à Paris, aura pour thème : « Syndromes douloureux chroniques en MPR ».

Retenez cette journée et venez nombreux car le programme, qui a été réalisé par les Drs Patricia Ribinik et Marc Genty, est très complet et fait intervenir des spécialistes de très haut niveau qui feront le point sur ce problème difficile.

Qu'ils en soient d'ores et déjà tous, coordinateurs et intervenants, chaleureusement remerciés.

Tout ce travail préparatoire de La Lettre, du site et de la Journée représente une somme de travail acharné et bénévole qui est élaborée par un petit noyau de membres actifs au cours de nos réunions mensuelles du bureau, tout ceci dans une ambiance à la fois sérieuse et conviviale.

Cette spécialité, cette Lettre, ce site, cette Journée sont les vôtres, et votre association, c'est tout cela.

Elle sert la promotion de notre spécialité, et sans vous, sans vos actions, vos écrits, vos récits, vos suggestions et bien sûr vos abonnements, elle ne serait rien.

Votre curiosité médicale et vos champs de compétences variés doivent vous mener à participer à la vie de la revue, du site et de la Journée. J'ai confiance en tous les membres de l'ANMSR et en l'avenir.

En attendant de nous retrouver bientôt lors de notre Journée du 24 mai au cours de laquelle aura lieu l'AG de notre association, au nom de tous les membres du bureau, je vous renouvelle tous mes vœux d'excellente année 2013. 